\title{
Of the People, For the People: Digital Literature Resource Knowledge Recommendation Based on User Cognition
}

Wen Lou, Hui Wang, and Jiangen He

\begin{abstract}
We attempt to improve user satisfaction with the effects of retrieval results and visual appearance by employing users' own information. User feedback on digital platforms has been proven to be one type of user cognition. Through conducting a digital literature resource organization model based on user cognition, our proposal improves both the content and presentation of retrieval systems. This paper takes Powell's City of Books as an example to describe the construction process of a knowledge network. The model consists of two parts. In the unstructured data part, synopses and reviews were recorded as representatives of user cognition. To build the resource category, linguistic and semantic analyses were used to analyze the concepts and the relationships among them. In the structural data part, the metadata of every book was linked with each other by informetrics relationships. The semantic resource was constructed to assist with building the knowledge network. We conducted a mock-up to compare the new category and knowledge-recommendation system with the current retrieval system. Thirty-nine subjects examined our mock-up and highly valued the differences we made for the improvements in retrieval and appearance. Knowledge recommendation based on user cognition was tested to be positive based on user feedback. There could be more research objects for digital resource knowledge recommendations based on user cognition.
\end{abstract}

\section{INTRODUCTION}

The concept of user cognition originates in cognitive psychology. This concept principally explores the human cognition process through information-processing methods. ${ }^{1}$ The concept characterizes a process in which a user obtains unknown information and knowledge through acquired information. As information-science workers, we may explore the psychological activities of users by analyzing their cognitive processes when they are using information services. ${ }^{2} \mathrm{~A}$ knowledge-recommendation service based on user cognition has become essential since it emphasizes facilitating collaborations between humans and computers and promotes the participation of users, which ultimately improves user satisfaction.

A knowledge-recommendation system is based on a combination of information organization, a retrieval system, and knowledge visualization. ${ }^{3}$ However, when exploring digital online literature resources, it is difficult to quickly and precisely find what we want because of the problem of information organization and retrieval. Most search results only display a one-by-one list view.

Wen Lou (wlou@infor.ecnu.edu.cn) is an assistant professor in the Faculty of Economics and Management, East China Normal University. Hui Wang (1830233606@qq.com) is a graduate student in the Faculty of Economics and Management, East China Normal University. Jiangen He (jiangen.he@drexel.edu) is a Doctoral Student in the College of Computing and Informatics, Drexel University. 
Thus, adding visualization techniques to an interface could improve user satisfaction.

Furthermore, the retrieval system and visualizations rely on information organization. Only if information is well designed can the retrieval system and visualization be useful.

Therefore, we attempt to improve retrieval efficiency by proposing a digital literature resource organization model based on user cognition to improve both the content and presentation of retrieval systems. Taking Powell's City of Books as an example, this paper proposes user feedback as first-hand user information. We will focus on (1) resource organizations based on user cognition and (2) new formats on search results based on knowledge recommendations. We will purposefully employ data from users' own information and give knowledge back to users in accordance with the quote "of the people, for the people."

\section{RELATED WORK}

\section{User Cognition and Measurement}

User cognition usually consists of a series of processes, including feeling, noticing, temporary memory, learning, thinking, and long-term memory. ${ }^{4}$ Feeling and noticing are at an inferior level, while learning, thinking, and memory are comparatively superior. Researchers have so far tried to identify user cognition processes by analyzing user needs. There are four levels of user needs according to Ma and Yang $^{5}$ (See Figure 1.) In turn, user interests normally reflect potential user needs. Users who retrieve information on their own show feeling needs. Users who give feedback show expression needs. Users who ask questions show knowledge needs, which is the highest level.

The methods to quantify user cognition require visible and measurable variables. Existing studies have commonly used website log analysis or user surveys. Website log analysis has been proven to be a solid data source to record and analyze both user interests and information needs. ${ }^{6}$ User surveys, including online questionnaires and face-to-face interviews, have been widely used to comprehend user feelings and user satisfaction. ${ }^{7}$ User surveys generally measure two kinds of relationship: between users and digital services and between users and the digital community. ${ }^{8}$ With a survey, we can make the most of statistics and assessment studies to analyze user satisfaction about an array of standards and systems of existing service platforms, service environments, service quality, and service personnel, which provides some references and suggestions for future study of user experience quality, platform elements, interaction process, and more. ${ }^{9}$ However, neither log data nor surveys can obtain first-hand user information in reallife settings. Eye tracking and the concept-map method can be used to understand user behavior in the course of user testing. ${ }^{10}$ However, these approaches are difficult to adapt to a large group of users.

Therefore, a linguistic-oriented review analysis has become an increasingly important method. User content, including reviews and tags, could be analyzed through text mining and become valuable data sources to learn their preferences for the product and service in the areas of electronic commerce and digital libraries. ${ }^{11}$ This type of data has been called "more than words." 12 


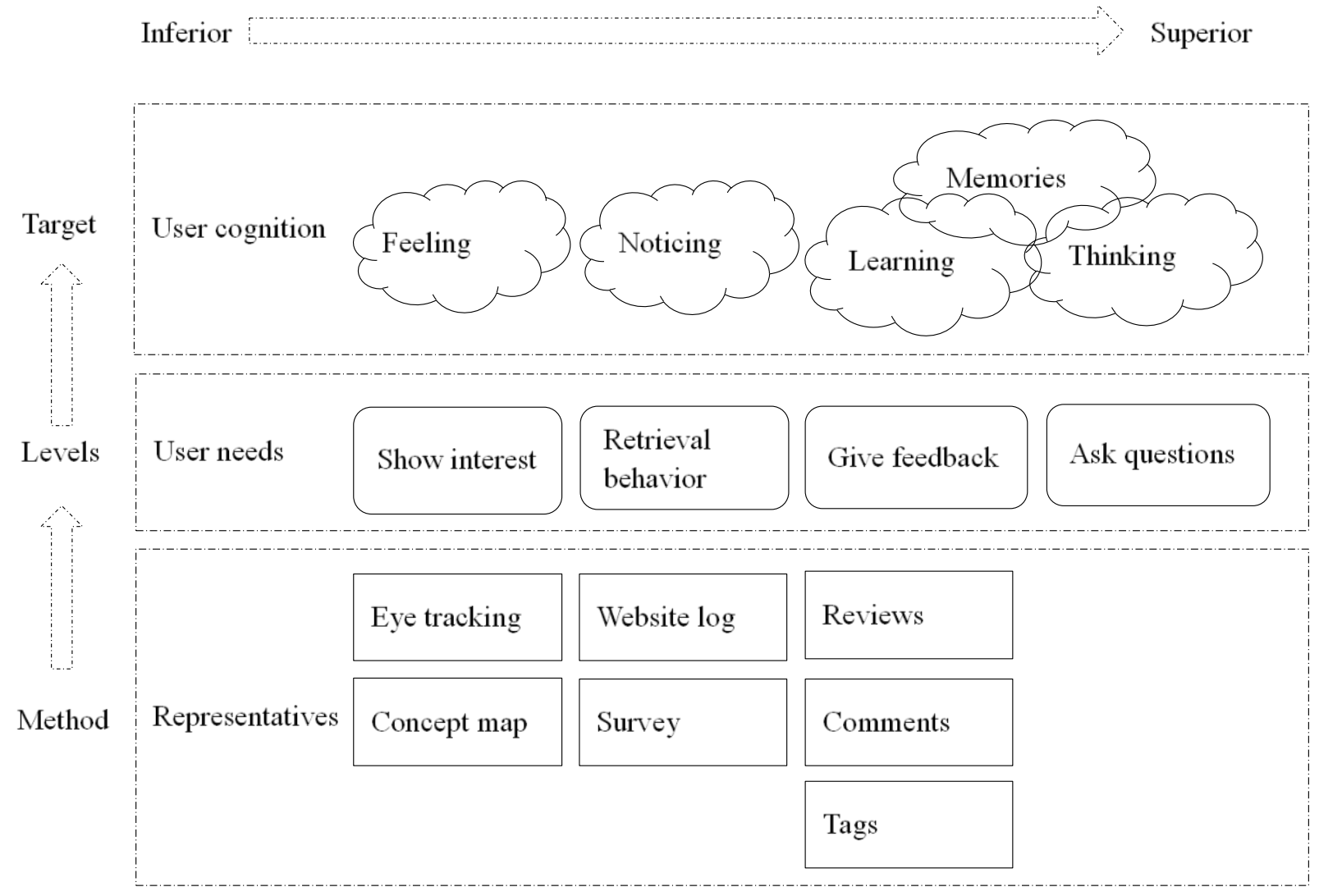

Figure 1. Understanding user cognition by analyzing user needs.

\section{User-Oriented Knowledge Service Model}

The user-oriented service model includes user demand, user cognition, and user information behavior. A service model based on user demand chiefly concentrates on the motives, habits, regularities, and purposes of user demand to identify the model of use demand so that the appropriate service is adopted. ${ }^{13}$ Service models based on user cognition attach importance to the process of user cognition, the influence that users are facing, ${ }^{14}$ and the change of library information services under the effects of series of cognitive processes (such as feeling, receiving, memorizing, and thinking). ${ }^{15} \mathrm{~A}$ service model based on user information behavior focuses on interactive behavior in the process of library information services that users participate in, such as interactions with academic librarians, knowledge platforms ${ }^{16}$ and others. Studies have paid more attention to the pre-process of the user-oriented service model, which analyzes information habits and user behaviors. ${ }^{17}$ Studies have also proposed frameworks of knowledge services, design innovations, ${ }^{18}$ or personalized systems and frames of the knowledge service model, but they have not succeeded in implementing or performing user testing.

\section{Knowledge Service System Construction}

Most studies of knowledge service system construction are in business areas. Numerous studies have explored knowledge-innovation systems for product services. ${ }^{19}$ Cheung et al. proposed a knowledge system to improve customer service. ${ }^{20}$ Vitharana, Jain, and Zahedi composed a knowledge repository to enhance the knowledge-analysis skills of business consultants. ${ }^{21}$ From 
the angle of user demand, Zhou analyzed the elements of service-platform construction and found that crucial platforms should serve knowledge service system construction. ${ }^{22}$ Scholars proposed basic models for knowledge management and knowledge sharing, but they did not simulate their applications. ${ }^{23}$ Knowledge management from the library-science perspective is very different from that in the business area. Library knowledge management usually refers to a digital library, especially a personal digital library. ${ }^{24}$ Others explore and attempt to construct a personalized knowledge service system, ${ }^{25}$ while fewer studies about system designs are based on the results of user surveys in accordance with documented surveys.

We rarely see a user-feedback study combined with the method of using users' own knowledge. Users themselves know what they desire. If user-oriented studies separate the system design from user-needs analysis or the other way around, the studies may miss the purpose. Therefore, we propose a resource-organization method based on users' own knowledge to close the distance between the users and the system.

\section{RESOURCE-ORGANIZATION MODEL BASED ON USER COGNITION}

There are normally two ways to construct a category system. One method gathers experts to determine categories and assign content to them; the category system comes first and the content second. The other method is to derive a category tree from the content itself, as we propose in this paper. In this way, the content takes priority over the categorization system. In this paper, we focus on this second way to organize resources and index content.

Resource organization requires a series of steps, including information processes, extraction, and organization. Figure 2 shows the resource-organization model based on user cognition. This model fits the needs of digital resources with comments and reviews. The model has two interrelated parts. One is for indexing the content, and the other is for knowledge recommendations.

For the first part, the model integrates all the comments and reviews of all literature in an area or the whole resource. The core concepts and the relationships among the concepts are extracted through natural language processing. The relationships between concepts are either subordination and correlation. A triple consists of two core concepts and their relationship. The triple set includes all triples.

Next, all books are indexed by taxonomy in the new category system. However, the indexing of every book is not based upon the traditional method, which is to manually determine each category by reading the literature. We use a method based on the books' content. While we are extracting the core concepts from all books we extract the core concepts from every book by the same semantic-analysis methods and build up triples for the individual book. Then the triples of this book can match the triple set in the new category system. Once a triple in a single book yields a maximum matching value, the core concepts in the triple set will be indexed as the keywords of the book. A few examples of the matching process will be discussed in the empirical study (in the section "Indexing Books").

The first part is about comments and reviews, which are unstructured data. The second part is to make use of structural data in the bibliography to build a semantic network. Structural data, including titles, keywords, authors, and publishers, is stored separately. We calculate the 
informetrics relationships among the entities. The relationships can be among different entities, such as between one author and another or between an author and a publisher. Then two entities and their relationship compose a triple. The components in triples are linked to each other, which makes them semantic resources. Furthermore, the keywords in structural data are not the original keywords before the new category system but are the modified keywords. Finally, the reindexed resources (books in the new category) and semantic resources (the triples from structural data) are both used to build the knowledge network.

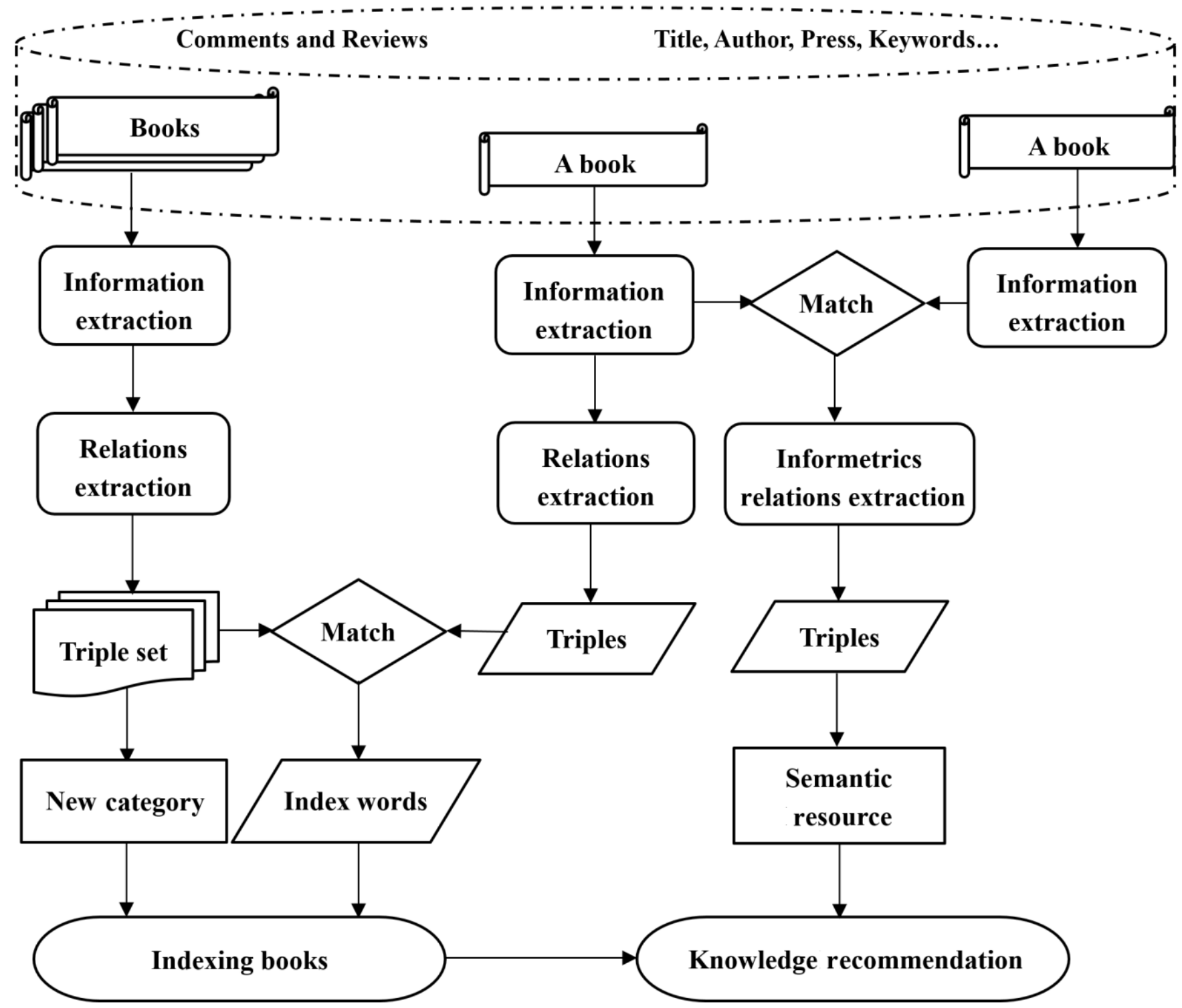

Figure 2. Resource-organization model based on user cognition.

However, why is it important to use both unstructured data and structural data? The reason is to complete the entire content of a literature resource. Neither of them can fully represent the whole semantics for a literature resource. Structural data lacks subjective content, and unstructured data lacks basic information. Thus, a full semantic network can be built using both kinds of data. 


\section{RESOURCE-ORGANIZATION EXPERIMENT}

\section{Object Selection}

Located in Portland, Oregon, Powell's City of Books (hereafter referred to as "Book City") is one of the largest bookstores in the United States, with 200 million books in its inventory. Book City caught our eyes for four reasons. (1) The comments and reviews of books on Book City's website are well constructed and plentiful. The National Geographic Channel established it as one of the ten best bookstores in the world. ${ }^{26}$ Atlantis Books, Pendulo, and Munro's Books are also on the list. Among these bookstores, only Book City and Munro's Books have indexed the information of comments and reviews. Since user reviews are fundamental to this study, we restricted ourselves to bookstores that provided user reviews. (2) We excluded libraries because literature resources have been well organized in libraries. It might not be necessary to reorganize them according to user cognition. However, we can put this topic in the future study. (3) Book City is a typical online bookstore that also has a physical bookstore. Unlike Amazon, Book City, Indigo, Barnes \& Noble, and Munro's Books have physical bookstores. However, they all have technological limitations on retrieval-system and taxonomical construction compared to Amazon. Thus, it is necessary to investigate these bookstores' online systems and optimize them. (4) The location was geographically convenient to the researchers. The authors are more familiar with Book City than other bookstores. Moreover, we plan on conducting a face-to-face interview for the user study. It is doable only if the authors can get to the bookstore and the users who live there. In all, we choose Book City as a representative object.

\section{Data Collection and Processing}

On December 22, 2015, we randomly selected the field "Cooking and Food" and downloaded bibliographic data for 462 new and old books that included title, picture, synopsis and review, ISBN, publication date, author, and keywords. In our previous work we described how metadata for all kinds of literature can be categorized into one of three types: structural data, semistructural data, and unstructured data. ${ }^{27}$ (See table 1).

Title, ISBN, date, publisher, and author are classified as structural data. Titles can be seen as structural data or unstructured data depending on the need. Titles will be considered as an indivisible entity in this paper as titles need to retain their original meanings. Keywords are considered as semistructural data for two reasons: (1) normally one book is indexed with multiple keywords, which are natural language; and (2) keywords are separated by punctuation. Each keyword can individually exist with its own meaning. However, in the current category system, keywords are the names of categories and subcategories. Since we are about to reorganize the category system, the current keywords will not be included in the following steps.

We use the field "Synopsis and Review" in the downloaded bibliographic records as the source of user cognition. Synopses and reviews are classified as unstructured data. All synopses and reviews of a single book are first incorporated into one paragraph, since some books contain more than one review.

Structural data will be stored for constructing a knowledge network. Unstructured data will be part-of-speech tagged and word segmented by the Stanford Segmenter. All the books' metadata are stored into the defined three data types and separate fields. Each field is linked by the ISBN as the primary key. 


\section{Category Organization}

First, the frequencies of words in all books are separately calculated after word segmenting so that core concepts are identified by the frequencies of words. In total, 29,370 words appeared 43,675 times, after excluding stop words. The 206 words in the sample that occurred more than 105 times appeared 34,944 times. This subset was defined as the core words according to the Pareto principle.

Table 1. Data Sample.

\begin{tabular}{|l|l|l|}
\hline Field & Content & \multirow{2}{*}{ Data type } \\
\hline Title & A Modern Way to Eat: 200+ Satisfying Vegetarian Recipes & \multirow{2}{*}{ Structural data } \\
\hline ISBN & 9781607748038 & \\
\hline Date & $04 / 21 / 2015$ & \multirow{2}{*}{ Semistructural data } \\
\hline Publisher & Ten Speed Press & \multirow{2}{*}{ Unstructured data } \\
\hline Author & Anna Jones & \\
\hline $\begin{array}{l}\text { KWnopsis } \\
\text { and } \\
\text { Review }\end{array}$ & $\begin{array}{l}\text { A beautifully photographed and modern vegetarian cookbook packed with } \\
\text { quick, healthy, and fresh recipes that explore the full breadth of vegetarian } \\
\text { ingredients_-grains, nuts, seeds, and seasonal vegetables_from Jamie } \\
\text { Oliver's London-based food stylist and writer Anna Jones. How we want to } \\
\text { eat is changing. More and more people cook without meat several nights a } \\
\text { week and are constantly seeking to ... }\end{array}$ & \\
\hline
\end{tabular}

We are inspired by Zhang et al., who described a linguistic-keywords-extraction method by defining multiple kinds of relationships among words. ${ }^{28}$ The relationships include direct relationship, indirect relationship, part-whole relationship, and related relationship.

- Direct relationship. Two core words have a relationship directly to each other.

- Indirect relationship. Two core words are related and linked by another word as a media.

- Part-whole relationship. The "is a" relation. One core word belongs to the other. It is the most common relationship in context.

- $\quad$ Related relationship. Two core words have no relationships but they both appear in a large context.

The first two relationships can be mixed with the second two relationships. For instance, a partwhole relationship can have either a direct relationship or an indirect relationship.

For this study, we combined every two core words into pairs for analysis. For example, the sentence "A picnic is a great escape from our day-to-day and a chance to turn a meal into something more festive and memorable" would result in several core-word pairs, including 
"Picnic" and "Meal," "Picnic" and "Festive," and "Meal" and "Festive." For "Picnic" and "Meal," there is an obvious part-whole relationship in this context. We observed all their relationships in all books and determined their relationship as a direct part-whole relationship because 67 percent of their relationships are part-whole relationship, 80 percent are direct relationship, and others are related relationship. This is the case when two core words are in the same sentence. For two words in different sentences but within one context, we define the words' relationship as a sentence relationship. For example, "Ingredient" and "Meat" in one review in table 1 have an indirect relationship because they are connected by other core concepts between them. Therefore, the relationship between "Ingredient" and "Meat" is an indirect part-whole one in this context. For other cases, two concepts are either related if they appear in the same context or are not related if they do not appear in the same review. Thus, all couples of concepts are calculated and stored as semantic triples.

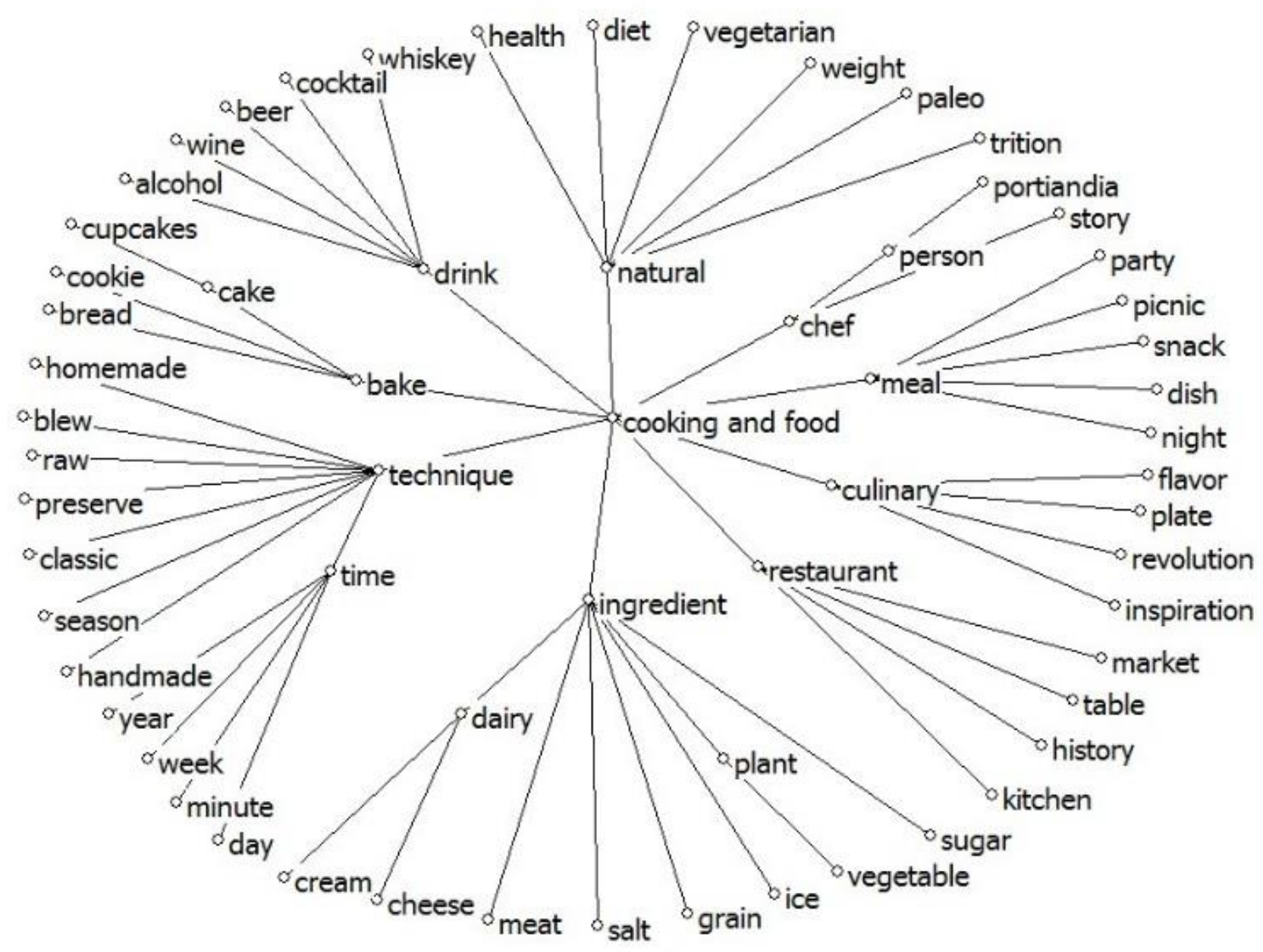

Figure 3. Parts of a modified category in "Cooking and Food" based on user cognition.

The next step is to build up a category tree (figure 4). A direct part-whole relationship is that between a parent class and child class. An indirect part-whole relationship is the relationship between a parent class and a grandchild class. A related relationship is the relationship between sibling classes. 
Compared to the modified category system (figure 3), the current hierarchical category system (figure 4) has two major issues. First, some categories' names are duplicated. For example, the child class "By Ingredient" contains "Fruit," "Fruits and Vegetables," and "Fruits, Vegetables, and Nuts." Second, there are categories without semantic meaning, such as "Oversized Books." These two problems brought out disorderly indexing and recalled many irrelevant results. For example, the system would let you refine your search first if you type one word in search box. However, refining is confusing by parent class and children class. Searching "diet" books as an example, the system suggests you refine your search from five subcategories of "Diet and Nutrition" under three different parent classes. However, the modified category system has avoided the duplicated keywords. Furthermore, the hierarchical system based on users' comments maintains meaning.

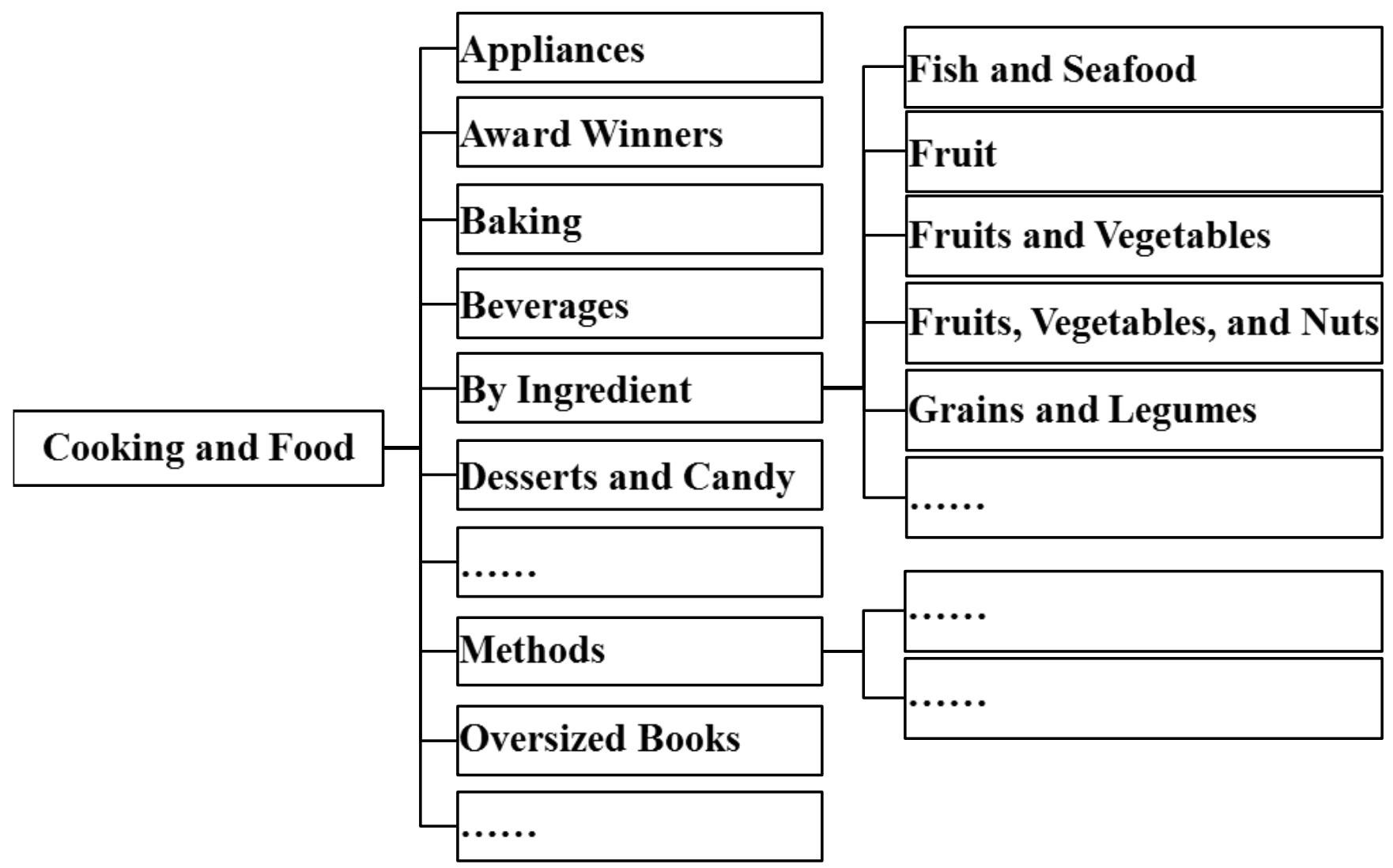

Figure 4. Parts of current category system in "Cooking and Food."

\section{Indexing Books}

We found that the list of keywords was confusing due to the inefficiency of the previous category system. It is necessary to re-index the keywords of each book based on the modified category system. We stand on the data-oriented indexing process. The method to detect the core concepts of each book is the same as that for all books in section 4.3. Taking the book A Modern Way to Eat as an example, triples are extracted from the book, including "grain-direct part whole-ingredient," "nut-direct part whole-ingredient," "vegetarian-related-health," and so on. Using all triples of the book to match with the triples set from all books in section 4.3, we index this book to categories by the best match parent class. In this case, 5 out of 9 triples of $A$ Modern Way to Eat are matched with the parent class "Ingredient." Another two are matched with "Natural" and "Technique," and 
the other two cannot correctly match with the triples set. Then, A Modern Way to Eat will be indexed with "Cooking and Food-Ingredient," "Cooking and Food-Natural," and "Cooking and Food-Technique."

\subsection{Semantic-Resource Construction}

The semantic resource is constructed based on structural data that was prepared at the beginning. The informetrics method (specifically co-word analysis) will be used to extract the precise relationship among the bibliography of books, as we previously proposed ${ }^{29} \mathrm{We}$ construct all structural data together and conduct co-words matrixes between each title, publisher, date, author, and keyword. For example, the author "Anna Jones" co-occurred with many keywords to varying degrees. The author co-occurred with the keyword "Natural" four times and "Person" seven times. According to Qiu and Lou, the precise relationship needs to be divided by the threshold and formatted as literal words. ${ }^{30}$ Therefore, among the degree of all relationships between "Anna Jones" and other keywords, the relationship between "Anna Jones" and "Natural" is highly correlated, and the relationship between "Anna Jones" and "Person" is extremely correlated. Triples are composed of two concepts and their relationships. Then a semantic resource is finally constructed that could be used for knowledge retrieval.

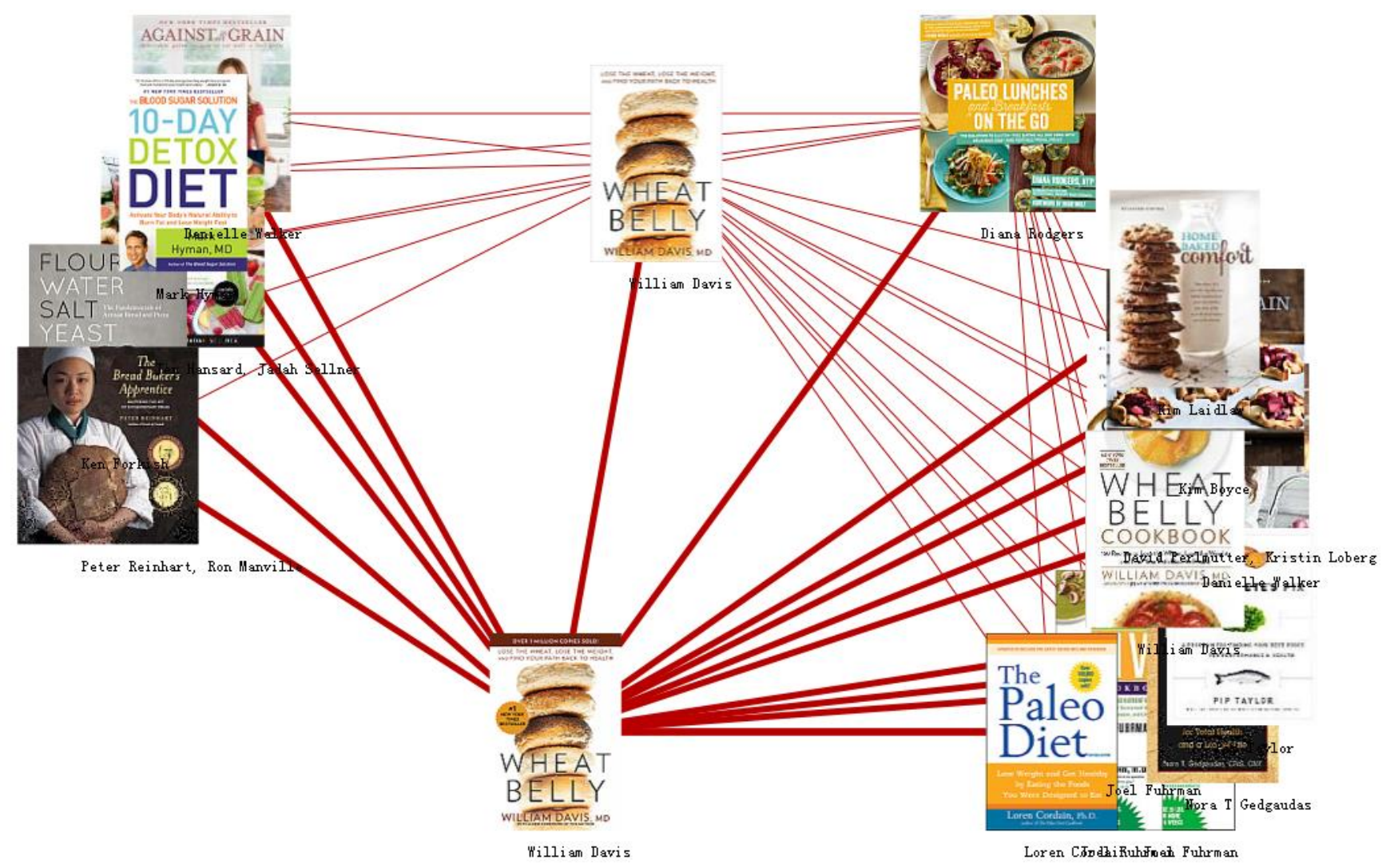

Figure 5. An example of the knowledge network.

Once the semantic resource is ready, the knowledge network is presentable. We adopted D3.js to display the knowledge network (figure 5). The net view automatically exhibits several books related with an author William Davis, which is placed in a conspicuous position on the screen. The forced map can be reformed when users drag any book with the mouse, which will be the noticeable center of other books. The network can connect with the database and the website. 


\section{USER-EXPERIENCE STUDY ON KNOWLEDGE DISPLAY AND RECOMMENDATION}

There are two common ways to evaluate a retrieval system. One is to test the statistic results, such as the recall and precision. The other is a user study. Since our aim is "of the people, for the people," we chose to conduct two user-experience studies over the statistical results. As such, we can obtain what users suggest and comment on our approach.

\section{User-Experience Study Design}

In February 2016, with the help of friends, we recruited volunteers by posting fliers in Portland, Oregon. fifty volunteers contacted us. Thirty-nine responses were received by the end of March 2016 because the other eleven volunteers were not able to enroll in the electronic test.

Since we needed to test the feasibility of both the new indexing category and the knowledge recommendation, we set up the user study into two parts, including the comparison of the simple retrieval and the knowledge recommendation.

First, we requested permission to use the data source and website frame from Book City. However, we cannot construct a new website for Book City due to intellectual-property issues. Therefore, we constructed a practical mock-up to guide users to simulate a retrieval experiment. Following the procedure of the user experience design, we chose MockingBot (https://mockingbot.com) as the mock-up builder. MockingBot allows the demo users to experience a vivid system that will be developed later. The mock-up supports every tag that can be linked with other pages so that subjects could click on the mock-up just as they would on a real website. The demo is expected to help us (1) examine whether our changes would meet the users' satisfaction and (2) gather information for a better design. Then we performed face-to-face, userguided interviews to first gain experience on the previous retrieval system and then compare them with our results. We concurrently recorded the answers and scores of users' feedback. In the following sections, we will describe the interview process and present the feedback results.

\section{Study 1: Comparison of Simple Retrieval}

First, subjects were asked to search related books written by "Michael Pollan" at Powells.com (figure 6). As such, all subjects used the search box based on their instincts. Then they were asked to find a new hardcover copy of a book named Cooked: A Natural History of Transformation. We paid attention to the ways that subjects located the target. Only five of them used keyboard shortcuts to find the target. However, thirteen subjects stated their concerns regarding the absence of refinement options. Furthermore, we noticed that six subjects swept (moused over) the refinement area and then decided to continue eye screening. In the meantime, we recorded the time they spent looking for the item. After they found the target, all subjects gave us a score from one to ten that represented their satisfaction with the current retrieval system. 


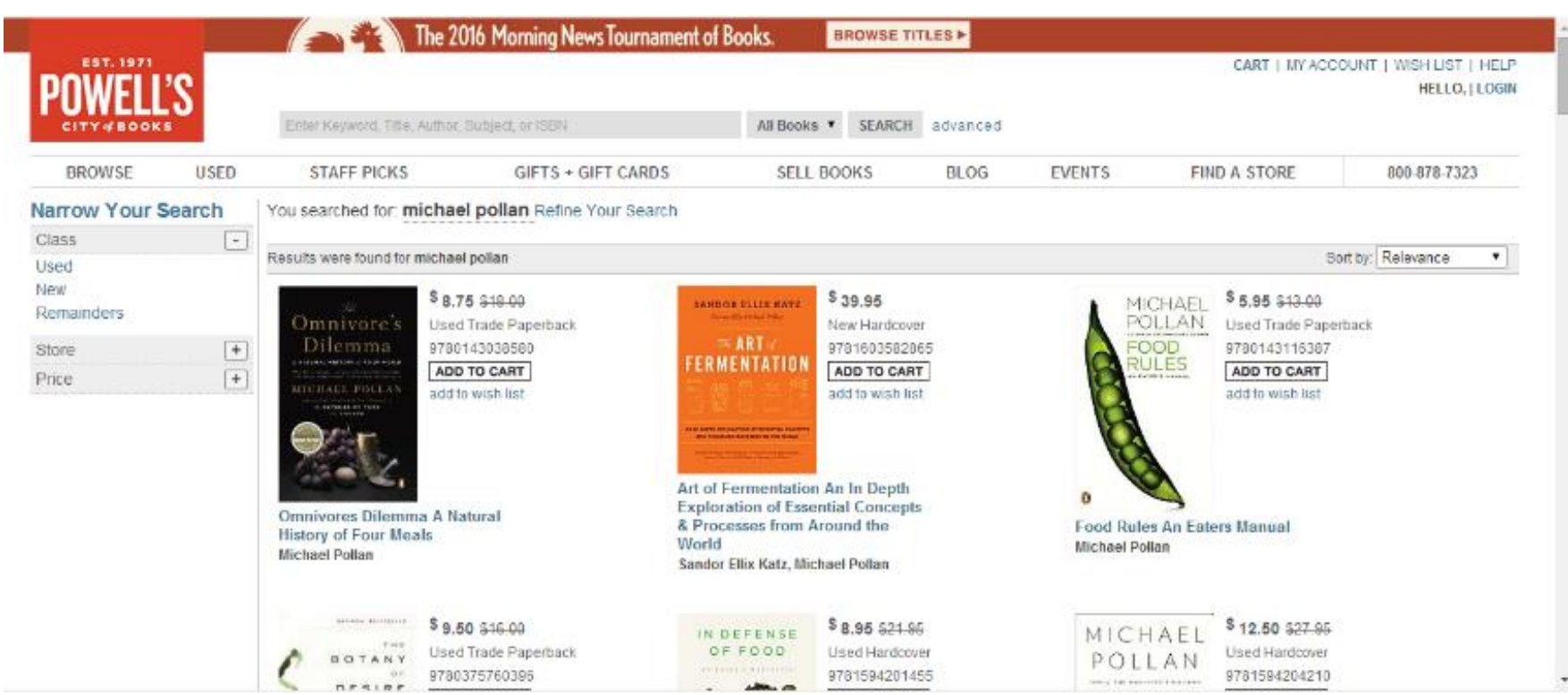

Figure 6. Screenshot of retrieval results in the current system.

In the comparison experiment, we placed our mock-up in front of subjects and conducted the same exam above. In the mock-up, we used the basic frame of the retrieval system but reframed the refinement area. In the new refinement area (figure 7), we added an optional box with refinement keywords in the left column to narrow the search scope. The logic of the refined keywords comes from the indexing category, as we mentioned in the section on the Indexing books. "Michael Pollan" was indexed in six categories: "Biographies," "Children's Books," "Cooking and Food," "Engineering Manufactures," "Hobby and Leisure," and "Gardening." Thus, when subjects clicked the "Cooking and Food" category, they can refine the results to only twelve books rather than the seventy books in the current system. Users can obtain accurate retrieval results faster. After the subjects completed their tasks, they gave us a score from one to ten representing their satisfaction with the modified retrieval system.

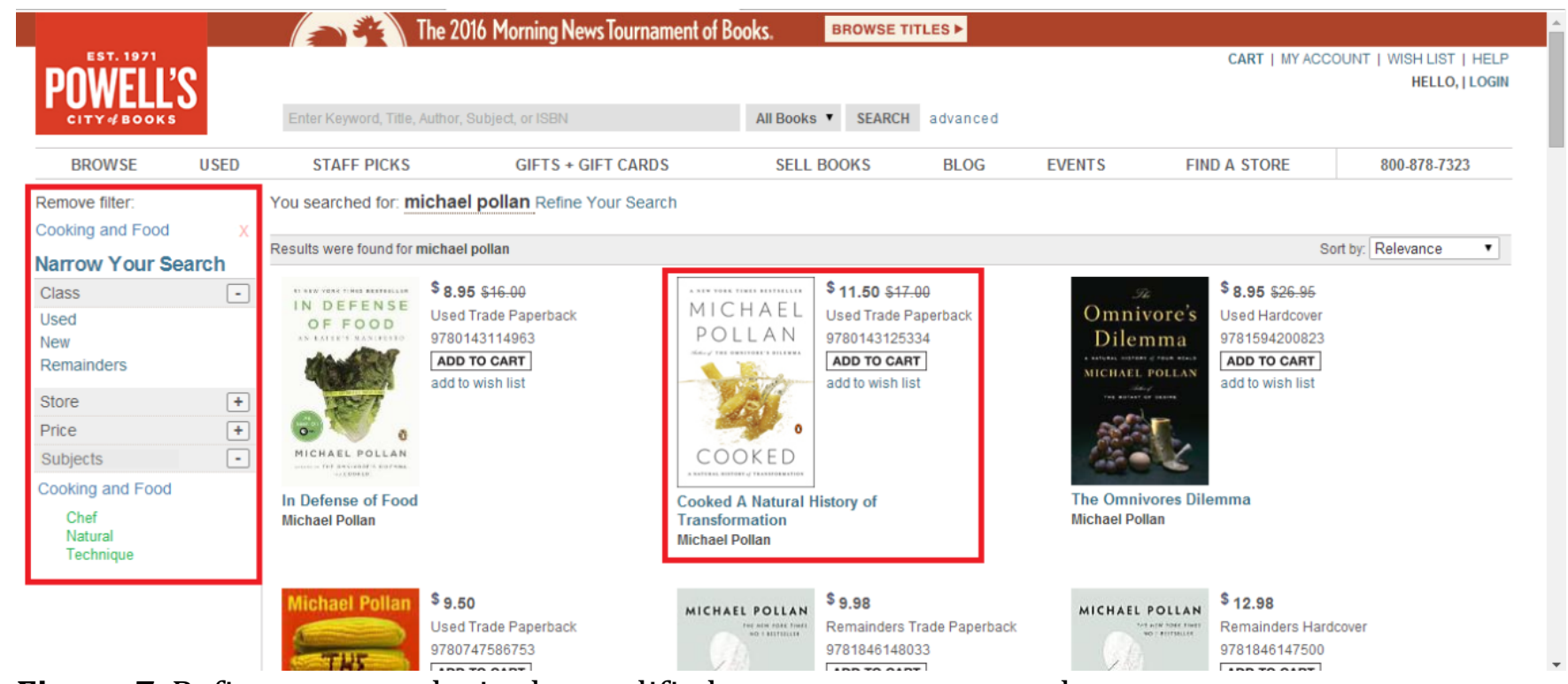

Figure 7. Refinement results in the modified category-system mock-up. 


\section{Study 2: Knowledge Recommendation}

In this experiment, we conducted two tests for two functions on knowledge visualization. One tested the preferences for the net view, and the other tested the preferences for the individual recommendation.

For the net view, we guided subjects to search for "William Davis" in the mock-up and reminded them to click the net view button after the system recalled a list view. Then, the subjects could see the net view results in figure 5. We recorded the scores that they gave for the net view.

As for the recommendation on individual books, we adopted multiple layers of associated retrieval results for every book. Users could click on one book and another related book would show in a new tab window. We asked subjects to conduct a new search for "William Davis." Then they could browse the website and freely click on any book. Once they clicked on Davis's book Wheat Belly: Lose the Wheat, Lose the Weight, and Find Your Path Back to Health, the first recommendation results popped up (figure 8). The recommendation results about wheat in the field of "Grain and Bread" showed up, including Good to the Grain: Baking with Whole Grain Flours and Bread Bakers Apprentice: Mastering the Art of Extraordinary Bread. Others about health and losing weight showed up also, such as Paleo Lunches and Breakfasts on the Go. All related books appeared because the first book is about both wheat and a healthy diet. A new window showing relevant authors and titles would pop up if the mouse glided over any picture. We asked the subjects about their thoughts on the new recommendation format and recorded the scores.

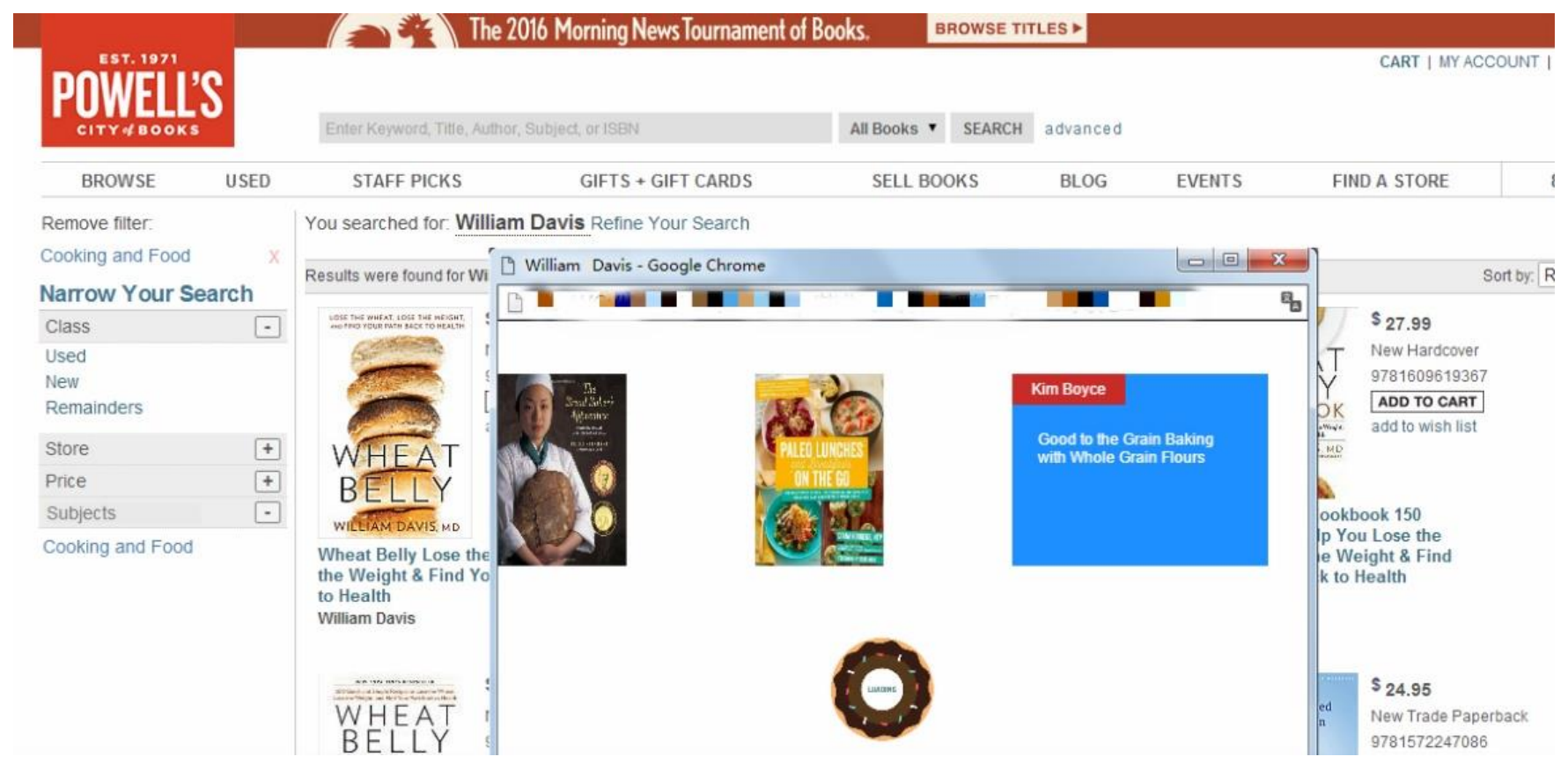

Figure 8. An example of knowledge recommendation.

\section{Users' Feedback}

As a result, knowledge organization and retrieval received a positive response (tables 2 and 3). First, subjects complained about the inefficiency of the current retrieval system in that it took so long to find one book without using shortcut keys (Ctrl-F). Three quarters of them were not satisfied with the original search style due to the search time length. However, 67 percent of the subjects gave a score of more than eight points for the refined search results of our new system. INFORMATION TECHNOLOGY AND LIBRARIES | SEPTEMBER 2018 
Only two of them thought that it was useless since they were the two users who only took ten seconds to target the exact result. Second, 67 percent and 74 percent of the subjects, respectively, thought that the knowledge recommendation and net view were useful and gave them six points. However, five subjects gave scores of one point because they maintained that it was not necessary to build a new viewer system.

Table 2. The time to find the exact result in the current system.

\begin{tabular}{|l|l|}
\hline Answers & \# of users \\
\hline Fewer than 10 seconds & 2 \\
\hline 10 to 30 seconds & 4 \\
\hline 30 seconds to 1 minute & 12 \\
\hline More than 1 minute & 21 \\
\hline
\end{tabular}

Table 3. Statistics of quantitative questions in the questionnaire.

\begin{tabular}{|c|c|c|c|c|c|c|c|c|c|c|c|}
\hline $\begin{array}{ll}\text { Questions } & \text { Score } \\
\end{array}$ & 10 & 9 & 8 & 7 & 6 & 5 & 4 & 3 & 2 & 1 & Total \\
\hline Satisfied with original results & 0 & 0 & 0 & 0 & 1 & 9 & 14 & 9 & 4 & 2 & 39 \\
\hline Preference of refined results & 2 & 10 & 14 & 6 & 5 & 0 & 0 & 0 & 0 & 0 & 37 \\
\hline Preference of results in net view & 1 & 8 & 10 & 6 & 4 & 1 & 2 & 3 & 1 & 3 & 39 \\
\hline Preference of knowledge recommendation & 3 & 6 & 4 & 8 & 5 & 6 & 0 & 3 & 1 & 2 & 38 \\
\hline
\end{tabular}

During the interview, subjects who gave scores of more than eight points spoke positively about the vivid visualization of the retrieval results, using words such as "innovative" and "creative." For instance, User 11 said, "Bravo changes for Powell, that'd be the most innovative experience for the locals." Among the subjects who gave scores of more than six points, the comments were mostly "interesting idea." For instance, User 17 commented, "This is an interesting idea to explore my knowledge. I had no idea Powell could do such an improvement." Some users offered suggestions to improve the system. For example, User 12 suggested that the system was not comprehensive enough to confidently assess whether the modified category system was better than the previous system. User 25 (a possible professional) was very concerned about the recall efficiency since the system might use many matching algorithms.

\section{DISCUSSION AND CONCLUSION}

In this paper, a digital literature resource organization model based on user cognition is proposed. This model aims to make users exert subjective initiative. We noticed a significant difference between the previous category system and the new system based on user cognition. Our aim, which was "of the people, for the people," was fulfilled. Taking Powell's City of Books as an example, it is purposeful to describe how to construct a knowledge network based on user cognition. The user experience study showed that this network implements an optimized exhibition of a digital-resource knowledge recommendation and knowledge retrieval. Although user cognition includes many other processes of user behavior, we only used the literal expression. It turned out to be a positive and possible way to reveal users' cognition. 
We find that there is much more space for the construction object of digital resource knowledge recommendation based on user cognition. For one, in this paper we only take the familiar Book City as a study object and books as experiment objects and determined favorable positive effects, which indicates that the digital resource knowledge link can be applied to physical libraries and bookstores or other types of literature. Even though libraries have well-developed taxonomy systems, they can be compared with or combined with new ideas. For another, users adore visual effects and user functions. The results show promise in actualizing improvements to Book City's website or even to other digital platforms. The concerns will be how to optimize the retrieval algorithm and reduce the time costs in the next study.

\section{ACKNOWLEDGEMENTS}

We thank Carolyn McKay and Powell's City of Books for such great help for the questionnaire networking and all participates for feedback. This work was supported by the National Social Science Foundation of China [grant number 17CTQ025].

\section{REFERENCES AND NOTES}

${ }^{1}$ Peter Carruthers, Stephen Stich, and Michael Siegal, The Cognitive Basis of Science (Cambridge: Cambridge University Press, 2002).

${ }^{2}$ Sophie Monchaux et al., "Query Strategies during Information Searching: Effects of Prior Domain Knowledge and Complexity of the Information Problems to Be Solved," Information Processing and Management 51, no. 5 (2015): 557-69, https://doi.org/10.1016/j.ipm.2015.05.004.

3 Hoill Jung and Kyungyong Chung, "Knowledge-Based Dietary Nutrition Recommendation for Obese Management," Information Technology and Management 17, no. 1 (2016): 29-42, https://doi.org/10.1007/s10799-015-0218-4.

4 Dandan Ma, Liren Gan, and Yonghua Cen, "Research on Influence of Individual Cognitive Preferences upon Their Acceptance for Knowledge Classification Recommendation Service," Journal of the China Society for Scientific and Technical Information 33, no. 7 (2014): 712-29.

${ }^{5}$ Haiqun Ma and Zhihe Yang, "Study on the Cognitive Model of Information Searchers from the Perspective of Neuro-Language Programming," Journal of Library Science in China 37, no. 3 (2011): 38-47.

${ }^{6}$ Paul Gooding, "Exploring the Information Behaviour of Users of Welsh Newspapers Online through Web Log Analysis," Journal of Documentation 72, no. 2 (2016): 232-46. https://doi.org/10.1108/JD-10-2014-0149.

${ }^{7}$ Munmun De Choudhury and Scott Counts, "Identifying Relevant Social Media Content : Leveraging Information Diversity and User Cognition," in 'HT11 Proceedings of the 22nd ACM Conference on Hypertext and Hypermedia (New York: ACM, 2011), 161-70, https://doi.org/10.1145/1995966.1995990; Carol Tenopir et al., "Academic Users' Interactions with ScienceDirect in Search Tasks: Affective and Cognitive Behaviors," Information Processing and Management 44, no. 1 (2008): 105-21, https://doi.org/10.1016/j.ipm.2006.10.007. 
${ }^{8}$ Young Han Bae, Jong Woo Jun, and Michelle Hough, "Uses and Gratifications of Digital Signage and Relationships with User Interface," Journal of International Consumer Marketing 28, no. 5 (2016): 323-31, https://doi.org/10.1080/08961530.2016.1189372.

9 Claude Sicotte et al., "Analysing User Satisfaction with the System in Use Prior to the Implementation of a New Electronic Inpatient Record," in Proceedings of the 12th World Congress on Health (Medical) Informatics; Building Sustainable Health Systems (Amsterdam: IOS Press, 2007), 1779-1784; Zhenzheng Qian et al., "SatiIndicator: Leveraging User Reviews to Evaluate User Satisfaction of SourceForge Projects," in Proceedings-International Computer Software and Applications Conference 1 (2016):93-102, https://doi.org/10.1109/COMPSAC.2016.183.

${ }^{10}$ Christina Merten and Cristina Conati, "Eye-Tracking to Model and Adapt to User Meta-Cognition in Intelligent Learning Environments," in Proceedings of the 11th International Conference on Intelligent User Interfaces-IUI '06 (New York: ACM, 2006), 39-46, https://doi.org/10.1145/1111449.1111465; Weidong Zhao, Ran Wu, and Haitao Liu, "Paper Recommendation Based on the Knowledge Gap between a Researcher's Background Knowledge and Research Target," Information Processing \& Management 52, no. 5 (2016): 976-88, https://doi.org/10.1016/j.ipm.2016.04.004.

${ }^{11}$ Haoran Xie et al., "Incorporating Sentiment into Tag-Based User Profiles and Resource Profiles for Personalized Search in Folksonomy," Information Processing and Management 52, no. 1 (2016): 61-72, https://doi.org/10.1016/j.ipm.2015.03.001; Francisco Villarroel Ordenes et al., "Analyzing Customer Experience Feedback Using Text Mining: A Linguistics-Based Approach," Journal of Service Research 17, no. 3 (2014): 278-95, https://doi.org/10.1177/1094670514524625; Yujong Hwang and Jaeseok Jeong, "Electronic Commerce and Online Consumer Behavior Research: A Literature Review," Information Development 32, no. 3 (2016): 377-88, https://doi.org/10.1177/0266666914551071.

12 Stephan Ludwig et al., "More Than Words: The Influence of Affective Content and Linguistic Style Matches in Online Reviews on Conversion Rates," Journal of Marketing 77, no. 1 (2012): 1-52, https://doi.org/10.1509/jm.11.0560.

13 Jun Yang and Yinglong Wang, "A New Framework Based on Cognitive Psychology for Knowledge Discovery," Journal of Software 8, no. 1 (2013): 47-54.

${ }^{14}$ Alan Baddeley, “On Applying Cognitive Psychology," British Journal of Psychology 104, no. 4 (2013): 443-56, https://doi.org/10.1111/bjop.12049.

${ }^{15}$ Aidan Moran, “Cognitive Psychology in Sport: Progress and Prospects," Psychology of Sport and Exercise 10, no. 4 (2009): 420-26, https://doi.org/10.1016/j.psychsport.2009.02.010.

${ }^{16}$ John Van De Pas, "A Framework for Public Information Services in the Twenty-First Century," New Library World 114, no. 1/2 (2013): 67-79, https://doi.org/10.1108/03074801311291974.

${ }^{17}$ Enrique Frias-Martinez, Sherry Y. Chen, and Xiaohui Liu, "Evaluation of a Personalized Digital Library Based on Cognitive Styles: Adaptivity vs. Adaptability," International Journal of 
Information Management 29, no. 1 (2009): 48-56, https://doi.org/10.1016/j.ijinfomgt.2008.01.012.

18 Shing Lee Chung et al., “An Integrated Framework for Managing Knowledge-Intensive Service Innovation," International Journal of Services Technology and Management 13, no. 1/2 (2010): 20, https://doi.org/10.1504/I]STM.2010.029669.

${ }^{19}$ Koteshwar Chirumalla, "Managing Knowledge for Product-Service System Innovation: The Role of Web 2.0 Technologies," Research-Technology Management 56, no. 2 (2013): 45-53, https://doi.org/10.5437/08956308X5602045; Koteshwar Chirumalla et al., "KnowledgeSharing Network for Product-Service System Development: Is It a Typical?," in International Conference on Industrial Product-Service Systems (2013): 109-14; Fumiya Akasaka et al., "Development of a Knowledge-Based Design Support System for Product-Service Systems," Computers in Industry 63, no. 4 (2012): 309-18, https://doi.org/10.1016/j.compind.2012.02.009.

20 C. F. Cheung et al., "A Multi-Perspective Knowledge-Based System for Customer Service Management," Expert Systems with Applications 24, no. 4 (2003): 457-70, https://doi.org/10.1016/S0957-4174(02)00193-8.

${ }^{21}$ Padmal Vitharana, Hemant Jain, and Fatemeh Zahedi, “A Knowledge Based Component/Service Repository to Enhance Analysts' Domain Knowledge for Requirements Analysis," Information and Management 49, no. 1 (2012): 24-35, https://doi.org/10.1016/j.im.2011.12.004.

${ }^{22}$ Baihai Zhou, "The Construction of Library Interdisciplinary Knowledge Sharing Service System," in 2014 11th International Conference on Service Systems and Service Management (ICSSSM), June 25-27, 2014, https://doi.org/10.1109/ICSSSM.2014.6874033.

${ }^{23}$ Rusli Abdullah, Zeti Darleena Eri, and Amir Mohamed Talib, “A Model of Knowledge Management System for Facilitating Knowledge as a Service (KaaS) in Cloud Computing Environment," 2011 International Conference on Research and Innovation in Information Systems, November 23-24, 2011, 1-4, https://doi.org/10.1109/ICRIIS.2011.6125691.

${ }^{24}$ Alan Smeaton and Jamie Callan, "Personalisation and Recommender Systems in Digital Libraries," International Journal on Digital Libraries 5, no. 4 (2005): 299-308, https://doi.org/10.1007/s00799-004-0100-1.

25 Yanwen Wu et al., "Research on Personalized Knowledge Service System in Community ELearning," Lecture Notes in Computer Science (Berlin: Springer, 2006), https://doi.org/10.1007/11736639 17; Shu-Chen Kao and ChienHsing Wu, "PIKIPDL. A Personalized Information and Knowledge Integration Platform for DL Service," Library Hi Tech 30, no. 3 (2012): 490-512, https://doi.org/10.1108/07378831211266627.

${ }^{26}$ National Geographic, Destinations of a Lifetime: 225 of the World's Most Amazing Places (Washington D.C.: National Geographic Society, 2016).

${ }^{27}$ Wen Lou and Junping Qiu, "Semantic Information Retrieval Research Based on Co-Occurrence Analysis," Online Information Review 38, no. 1 (January 8, 2014): 4-23, 
https://doi.org/10.1108/OIR-11-2012-0203; Junping Qiu and Wen Lou, "Constructing an Information Science Resource Ontology Based on the Chinese Social Science Citation Index," Aslib Journal of Information Management 66, no. 2 (March 10, 2014): 202-18, https://doi.org/10.1108/AJIM-10-2013-0114; Fan Yu, Junping Qiu, and Wen Lou, "Library Resources Semantization Based on Resource Ontology," Electronic Library 32, no. 3 (2014): 322-40, https://doi.org/10.1108/EL-05-2012-0056.

${ }^{28}$ Lei Zhang et al., "Extracting and Ranking Product Features in Opinion Documents," in International Conference on Computational Linguistics (2010): 1462-70.

${ }^{29}$ Lou and Qiu, "Semantic Information Retrieval Research," 4; Qiu and Lou, "Constructing an Information Science Resource Ontology," 202; Yu, Qiu, and Lou, "Library Resources Semantization," 322.

${ }^{30}$ Qiu and Lou, “Constructing an Information Science Resource Ontology," 202. 